\title{
Experimental Research on the Deformation and Failure of Concrete Under Biaxial Loading
}

\author{
Lu Mingfi ${ }^{l, 2}$, Bai Yilong ${ }^{l, *}$ \\ ${ }^{1}$ LNM, Institute of Mechanics, Chinese Academy of Sciences \\ Beijing, China \\ ${ }^{2}$ Graduate School of Chinese Academy of Sciences \\ Beijing, China
}

\begin{abstract}
In this paper, the biaxial loading experiment for concrete is performed. The boundary displacements of the specimen in two directions, as the governing variables, maintain certain ratios. Two failure modes are found, catastrophic rupture and gradual failure. The biaxial loads drop simultaneously in catastrophic rupture. And the fluctuation value of deformation rise rapidly before the catastrophic rupture occurs. This reveals a sign of localization under biaxial loading.
\end{abstract}

Catastrophic rupture, Progressive failure, Biaxial loads, Fluctuation (key words)

\section{I. 前言}

混凝土作为现代社会中主要的建筑材料之一, 对于社 会发展起着重要的作用[1], 其力学性能及失效问题备受研 究者们关注[2,7]。

混凝土由水泥、砂浆、石子等混合而成, 具有强度非 均匀性以及空间无序性的特点。针对这一类介质, 白以 龙、夏蒙棼等基于统计细观损伤力学, 从理论模型推导出 局部化触发灾变的条件, 发现灾变前损伤演化呈现幂律奇 异性的共性特征[8-10]。许向红、郝圣旺[11,12]等对岩石 的单轴实验结果表明, 即便对于初始宏观参数相近的试 样, 它们的载荷峰值和破坏灾变点差异很大[11], 这给用 宏观强度作为破坏指标带来了局限性; 另一方面, 对试样 表面的变形同步观测中发现, 灾变发生前试样的变形趋于 局部化, 利用变形观测的局部化尺度对灾变点的预测得到 了较为满意的结果, 同时也验证了灾变破坏的幂律特征, 为灾变破坏预测提供了参考途径[12]。

但是在实际的工程应用中, 混凝土一般处于复杂应力 状态, 仅限于单轴的结果还不能满足实际需求。为了研究 混凝土在复杂应力下的变形与破坏的特征, 我们进行了混 凝土双轴加载实验, 分析了混凝土双轴加载的破坏方式, 以及加载过程中变形规律。

\section{II. 实验方法}

\section{A. 试样制备}

本实验采用的试样为 $\mathrm{C} 30$ 素混凝土, 石子骨料, 粒 径为 $5-10 \mathrm{~mm}$, 胶凝材料为普通硅酸盐水泥 (浅野 P.O42.5R)。配合比为 $\left(1 \mathrm{~m}^{3}\right)$ : 石子: $1100 \mathrm{Kg}$, 砂石: $740 \mathrm{Kg}$, 水泥: $360 \mathrm{Kg}$, 水: $180 \mathrm{Kg}$ 。 28 天标准养护, 机 械振捣, 人工浇注。试样尺寸 $150 \times 150 \times 50\left(\mathrm{~mm}^{3}\right)$ 。

\section{B. 实验设置}

本实验采用的试验机为 INSTRON8506 四立柱液压伺 服系统。以试样边界变形为控制量进行加载, 并且令水平 与坚向位移的加载比为 $U_{y}: U_{x}=-1:-\alpha$ (这里负号代表 压缩), 分别进行了 $\alpha=0.1,0.3,0.7,1$ 的双轴压缩实验。 试验机压头与试样之间搁置减摩层, 以消除试样与压头之 间的摩擦力。采用准静态加载, $y$ 方向的压头行走速率为 每分钟 $0.027 \mathrm{~mm}$ 。
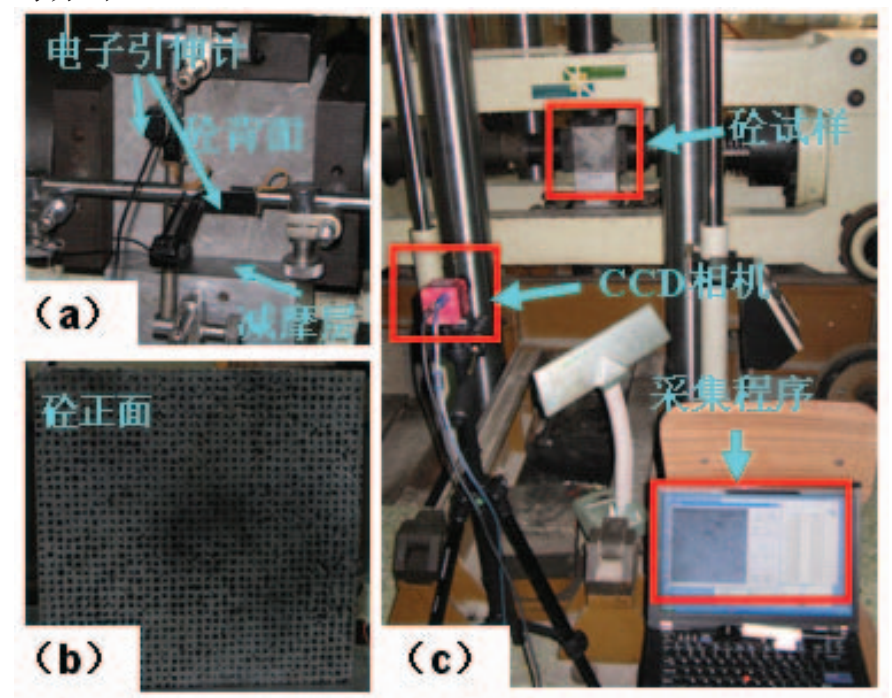

图 1. 实验观测布置。（a）试样背面电子引伸计及减 摩层, （b）. 试样表面散斑喷制, （c）CCD 相机布置与 图像采集。

实验过程中, 试验机系统通过背面的电子引伸计记录 试样在双轴加载方向的整体位移 $U_{x}, U_{y}$ 并返回试验机系统 进行闭合回路控制加载, 见图 1（a）。通过载荷传感器记 录加载力 $F_{x}, F_{y}$ 。在样品的自由表面（正面）喷制了人工 散斑, 见图 1 (b)。用 CCD 相机拍摄试样在加载过程中 自由表面的人工散斑图像序列, 见图 1（c）, 可以通过数 字散斑相关方法（DSCM）计算加载过程中的表面位移, 用于对表面变形场的分析。

"To whom correspondence should be addressed. E-mail: baiyl@Inm.imech.ac.cn 
III. 实验结果

实验得到了混凝土试样双轴加载的双轴载荷位移曲 线, 以及加载过程中 CCD 拍摄的试样表面散斑图像序 列。

\section{A. 破坏模式}

图 2 分别给出 $\alpha=0.1,1$ 两种加载比下的两个试样的 载荷位移曲线。

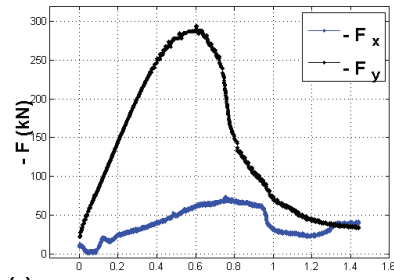

(a)

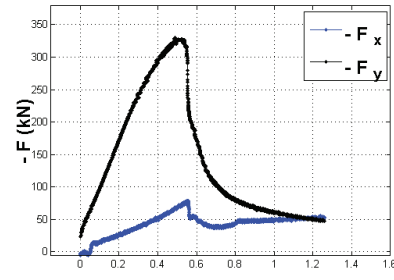

(b)

$-\mathrm{U}_{\mathrm{y}}(\mathrm{mm}), \alpha=0.1$
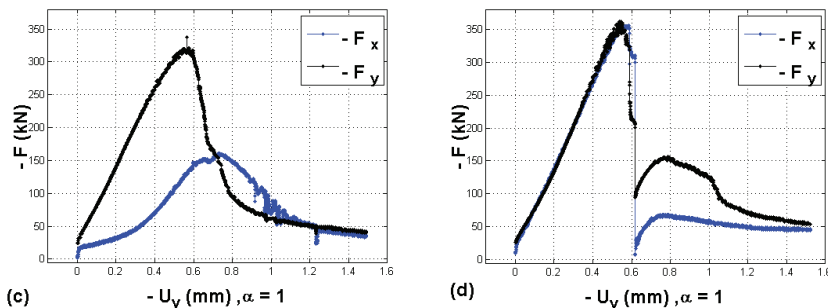

(d)

图 2. 加载比 $\alpha$ 为 0.1 的双轴载荷位移曲线（a）（b），以 及加载比 $\alpha$ 为 1 的双轴载荷位移曲线（c）（d）。

实验结果表明在所得到的 $\left(F_{x}, F_{y}\right) \sim U_{y}$ 关系中, 某些 试样的载荷位移曲线表现出准连续的关系如图 1（a） (c), 而某些试样的载荷值在峰值后, 双轴载荷同时迅 速地失去承载力, 如图 1 (b) (d), 为了分析出现这种 现象的原因, 在有限的加载控制量区间内 $\left[U_{y}, U_{y}+\Delta U_{y}\right]$ 考察载荷值的最大变化 $\Delta F_{y}$ 见图 3 , 这里区间间隔取为 $\Delta U_{y}=0.002 \mathrm{~mm}$, 约为 4 个加载步。在实验中, 因试验 机自身控制以及系统误差等原因，所测量的数据浮动应当 在一个相对稳定的范围内, 在图 3 中这种浮动长期保持在 $-11 \mathrm{kN}$ 与 $11 \mathrm{kN}$ 之间。然而图 3（b）中, 试样的载荷在峰 值之后出现异常大的下降, 最大幅度达到了- $45 \mathrm{KN}$, 远超 过了相对稳定情形的范围。可以认为, 这种载荷的突降不 能归结为随机涨落, 而是试样自身力学性能的反映。这与 郝等对单轴情形的研究结果有相似之处。郝等通过一维的 单轴解析模型分析，发现，由于局部化尺度的不同，损伤 演化会出现准连续和灾变两种破坏模式, 并在岩石和混凝 土的单轴加载实验中得到了验证。为此, 本实验分析中将 这类载荷突降幅度超过试验机正常浮动范围的破坏归类为 混凝土双轴加载下的灾变破坏, 而未出现超过试验机正常 浮动范围的破坏归类为渐进破坏。

根据上述的实验判据, 将不同加载比 $\alpha$ 下, 共 18 个 双轴压缩试样的破坏类型归类如表 1 。
表 1 双轴压缩加载的破坏类型分类

\begin{tabular}{lllll}
\hline 加载比 $\alpha$ & 0.1 & 0.3 & 0.7 & 1 \\
\hline 灾变破坏数 & 3 & 4 & 3 & 2 \\
渐进破坏数 & 3 & 1 & 1 & 1 \\
总数 & 6 & 5 & 4 & 3 \\
\hline
\end{tabular}
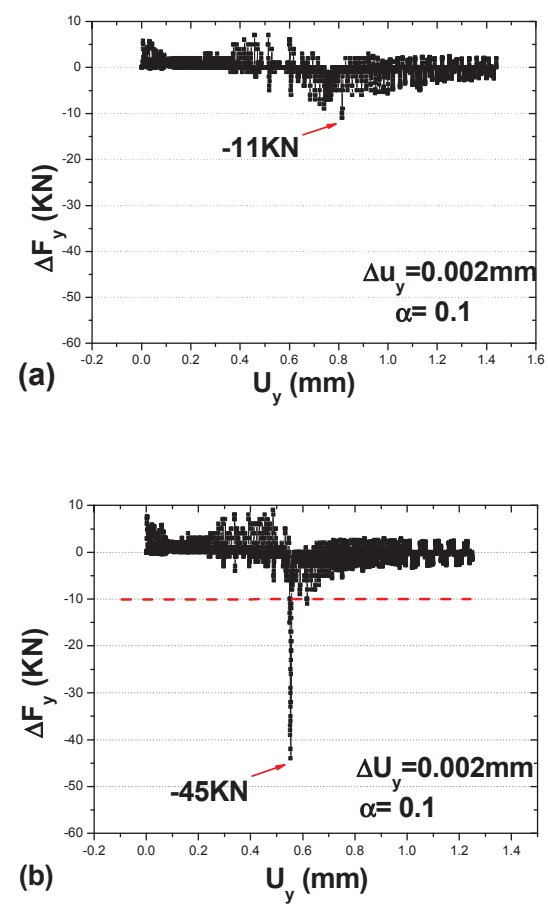

图 3 加载比 $\alpha=0.1$ (a) $\alpha=1$ (b)的 $\Delta F_{y} \sim U_{y}$ 关系

\section{B. 表面变形场演化特征的跨尺度分析}

混凝土等非均匀介质的破坏对试样内部损伤场的细节 具有敏感的依赖性, 而样本的表面变形的演化在一定程度 上反映了试样内部损伤场的变化, 为此, 本文通过 $\mathrm{CCD}$ 相机记录了加载过程中试样表面散斑图像, 利用数字散斑 相关方法 (DSCM) 计算试样表面的位移 $[13,14]$, 试图从 试样的细节分析最终破坏的规律。
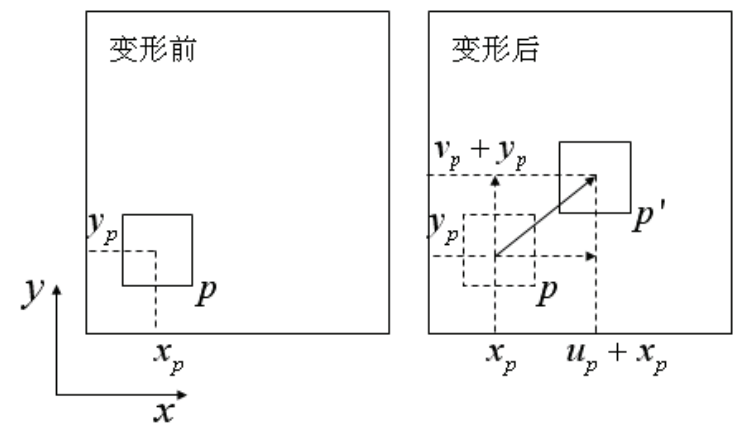

图 4. 变形前后图像位移计算示意图

如图 4 所示, 以 $p$ 点为中心的区域, 经过变形产生 了相应的位移, 按 (1) 式定义相关系数 


$$
C\left(M_{1}, M_{2}\right)=\frac{\operatorname{Cov}\left(M_{1}, M_{2}\right)}{\sqrt{D\left(M_{1}\right) \times D\left(M_{2}\right)}}
$$

式中:

$M_{1}$ 为变形前待求位移区域的图像灰度值矩阵。

$M_{2}$ 为变形后搜索区域的图像灰度值矩阵。

$\operatorname{Cov}\left(M_{1}, M_{2}\right)$ 为 $M_{1}$ 与 $M_{2}$ 的协方差。

$D\left(M_{1}\right), D\left(M_{2}\right)$ 分别为 $M_{1}$ 与 $M_{2}$ 的方差。

在变形后的图像中 $p$ 点周围的区域，寻找相关系数 值最大时对应的区域中心位置 $p^{\prime}$ 点, 认为 $p^{\prime}$ 点相对于 $p$ 点的位移 $\left(u_{p}, v_{p}\right)$ 为以 $p$ 点为中心的区域经过变形产生的 位移。

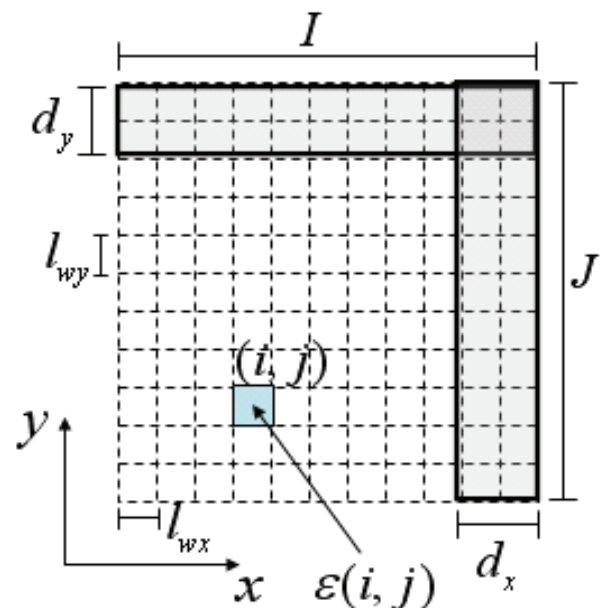

图 5. 对数字图像划分的相关窗口 (细虚线) 以及粗 粒化分析窗口（粗实线）。

将初始图像划分为 $I \times J$ 个用于计算位移的相关窗 口, 如图 5 所示, 利用数字散斑相关方法, 计算加载过程 中的图像相对于初始图像的累积位移 $(u, v)$, 再用 (2) 式的差分方法计算应变

$$
\begin{aligned}
& \varepsilon_{x}(i, j)=\frac{u(i+1, j)-u(i-1, j)}{2 l_{w x}} \\
& \varepsilon_{y}(i, j)=\frac{v(i+1, j)-v(i-1, j)}{2 l_{w y}} \\
& \gamma_{x y}(i, j)=\frac{u(i, j+1)-u(i, j-1)}{2 l_{w y}}+\frac{v(i+1, j)-v(i-1, j)}{2 l_{w x}}
\end{aligned}
$$

式中:

$(i, j)$ 分别为划分的区域在 $x, y$ 方向的位置。

$l_{w x}, l_{w y}$ 分别为相邻计算区域中心在 $x, y$ 方向的距离。

混凝土由多种成份组成，强度的非均匀性以及空间分 布无序性, 导致了试样内变形响应的非均匀性。为分析非 均匀性变形的统计特征, 分别从 $x, y$ 方向选取了粗粒化窗
口, 如图 5 所示, 对 (2) 中的三个应变分量 (以 $\varepsilon$ 代

替）统计 $d_{y} \times I$ 个粗粒化窗口内的平均应变:

$$
<\varepsilon>^{x}\left(j^{\prime}, d_{y}\right)=\frac{1}{I \times d_{y}} \sum_{i=1, j=1}^{i=I, j=d_{y}} \mathcal{E}(i, j)
$$

得到一定的 $d_{y}$ 下 $J^{\prime}$ 个粗粒化窗口的应变平均值。

以及 $d_{x} \times J$ 个粗粒化窗口内的平均应变:

$<\varepsilon>^{y}\left(i^{\prime}, d_{x}\right)=\frac{1}{J \times d_{x}} \sum_{i=1, j=1}^{i=d_{x}, j=J} \varepsilon(i, j)$

得到一定的 $d_{x}$ 下 $I^{\prime}$ 个粗粒化窗口应变平均值。

整个表面的平均应变为:

$<\varepsilon>=\frac{1}{I \times J} \sum_{i=1, j=1}^{i=I, j=J} \varepsilon(i, j)$

定义应变分量 $\mathcal{E}$ 在 $x, y$ 方向的涨落分别为:

$$
\begin{aligned}
& H^{x}\left(\varepsilon, d_{x}\right)=\frac{1}{I^{\prime}} \sum_{i^{\prime}=1}^{i^{\prime}=I^{\prime}}\left(<\varepsilon>^{y}\left(i^{\prime}, d_{x}\right)-<\varepsilon>\right)^{2} \\
& H^{y}\left(\varepsilon, d_{y}\right)=\frac{1}{J^{\prime}} \sum_{j^{\prime}=1}^{j^{\prime}=J^{\prime}}\left(<\varepsilon>^{x}\left(j^{\prime}, d_{y}\right)-<\varepsilon>\right)^{2}
\end{aligned}
$$

应变分量 $\varepsilon_{x}$ 在 $x$ 方向的粗粒化窗口内的平均值 $\left\langle\varepsilon_{x}\right\rangle^{x}$ 为试样整体应变, 应变分量 $\varepsilon_{x}$ 在 $y$ 方向的涨落 $H^{y}\left(\varepsilon_{x}\right)$ 并不能反映试样内的非均匀变形特征, 因此分析 了加载过程中 $H^{x}\left(\varepsilon_{x}\right), H^{y}\left(\varepsilon_{y}\right)$ 随着对应所选粗粒化窗 口尺度 $d_{x} / I, d_{y} / J$ 的演化过程。
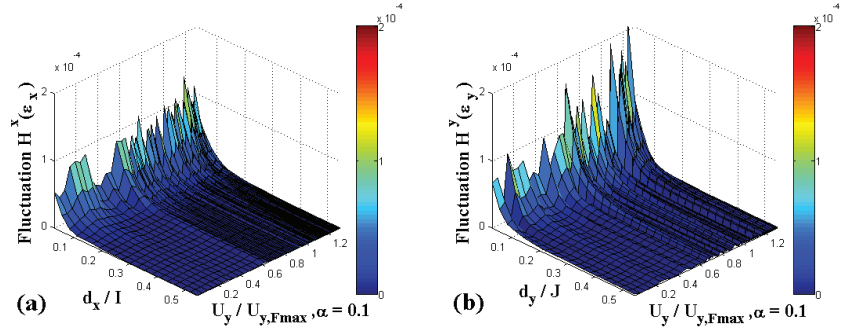

图 6. $\alpha=0.1$ 双轴压缩, 准连续破坏试样在加载过程 $\left[0,1.3 U_{y, F \max }\right]$ 的涨落值演化, (a) $H^{x}\left(\varepsilon_{x}\right)$ 的演化, (b) $H^{y}\left(\varepsilon_{y}\right)$ 的演化

图 6 给出 $\alpha=0.1$ 加载情况下, 准连续破坏试样的涨落 值 $H^{x}\left(\varepsilon_{x}\right), H^{y}\left(\varepsilon_{y}\right)$ 演化, 其中 $U_{y, F \text { max }}$ 是 $y$ 方向载荷 峰值处的边界位移, 由于 $y$ 方向载荷峰值后, 试样表层发 生脱落, 不便于对全场的统计分析, 为此分析了涨落值在 
加载区间 $\left[0,1.3 U_{y, F \max }\right]$ 内的演化, 结果表明在准连续破 坏情形, 试样表面发生脱落之前, 正应变 $\varepsilon_{x}, \mathcal{E}_{y}$ 的涨落 值没有出现显著的升高。
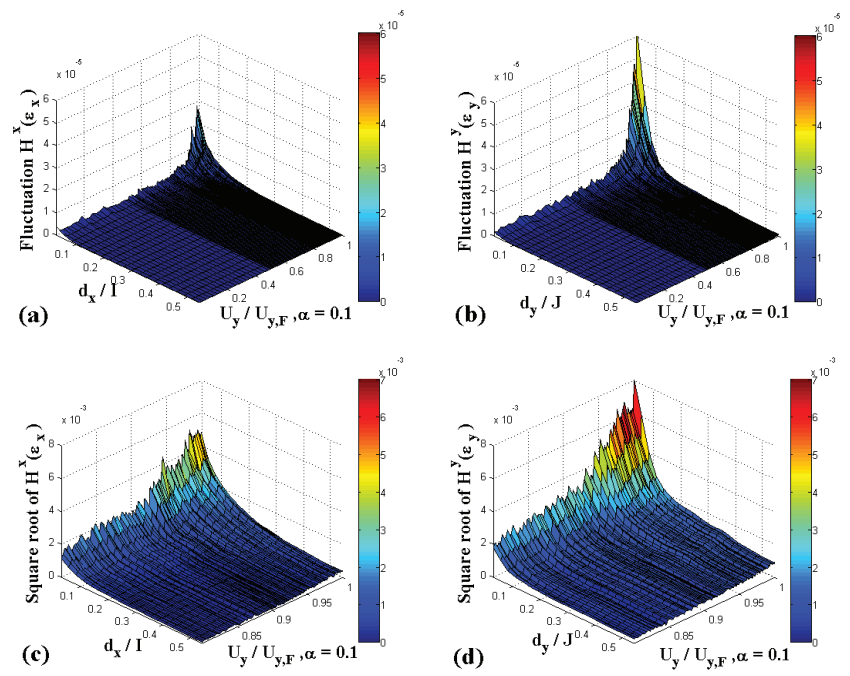

图 7. $\alpha=0.1$ 双轴压缩, 灾变破坏情形, 试样在加载 过程 中的涨落值演化, (a) $H^{x}\left(\varepsilon_{x}\right)$ 的演化,

(b) $H^{y}\left(\varepsilon_{y}\right)$ 的演化, (c) $\sqrt{H^{x}\left(\varepsilon_{x}\right)}$ 的演化,

(d) $\sqrt{H^{y}\left(\varepsilon_{y}\right)}$ 的演化

图 7 给出 $\alpha=0.1$ 加载情况下, 灾变破坏试样的涨落值 $H^{x}\left(\varepsilon_{x}\right), H^{y}\left(\varepsilon_{y}\right)$ 演化, 其中 $U_{y, F}$ 为载荷发生灾变突 跳前一点的边界位移, 结果发现在灾变之前正应变 $\varepsilon_{x}$, $\varepsilon_{y}$ 的涨落值显著升高, 且 $H^{y}\left(\varepsilon_{y}\right)$ 显著高于 $H^{x}\left(\varepsilon_{x}\right)$, 从 $\left[0.6 U_{y, F}, U_{y, F}\right]$ 区间内 $\sqrt{H^{x}\left(\varepsilon_{x}\right)}$ 和 $\sqrt{H^{y}\left(\varepsilon_{y}\right)}$ 的演化可 以看出, 在 $d_{x} / I, d_{y} / J$ 小于 0.4 尺度的范围内, 灾变 前涨落明显升高, 这种跨尺度涨落增强现象反映了灾变前 出现了相应特征尺度的局部化现象。

\section{IV. 结论}

本文通过混凝土双轴加载同步观测实验, 看到了两种 破坏模式: 准连续模式和灾变模式, 在灾变模式的破坏 中, 双轴载荷的突跳具有同步性, 这可能是混凝土失效的 一种普遍现象。混凝土在加载过程中的变形具有跨尺度涨 落的特征, 对于发生灾变破坏的试样, 变形的非均匀性在
灾变前显著升高, 并且在一定的尺度范围内, 变形的非均 匀性显著升高, 这是混凝土双轴加载下出现局部化的反 映。

\section{致谢}

感谢国家自然科学基金（90715001，10721202， 10972218 ）和 973 计划 2007CB814803 的资助。

\section{References}

[1] S. H. Guo, The Research Progress in Failure Theory of Concrete. Advances in Mechanics, Vol 23, pp. 520 529,1993. (In Chinese).

[2] H. L. Wang and Y. P. Song, Behaviour of mass concrete under biaxial compression-tension and triaxial compression-compression-tension. Materials and Structures, Vol 42, pp. 241 249, 2009.

[3] Li X. F., Tian J., Huang H. L., Zhang H., and Zhang C. Numerical Experiment on Concrete Specimen under Biaxial Stress. China Harbour Engineering, Vol 159, pp. 18 21, 2009. (In Chinese)

[4] W. Suaris and J. G. M. Vanmier, Acoustic emission source characterization in concrete under biaxial loading. Materials and Structures, Vol 28, pp. 444 449, 1995.

[5] H. S. Shang and Y. P. Song, Experimental study of strength and deformation of plain concrete under biaxial compression after freezing and thawing cycles. Cement and Concrete Research, Vol 36, pp. 1857 1864, 2006.

[6] E. K. Tschegg and M. Elser, Mode I fracture behaviour of concrete under biaxial loading. Journal of Materials Science, Vol 30, pp. 235 242,1995.

[7] X. Y. Tao and David V. Phillips, A simplified isotropic damage model for concrete under bi-axial stress states. Cement \& Concrete Composites, Vol 27, pp. 716 726, 2005.

[8] Y. L. Bai, C. S. Lu, F. J. Ke, and M. F. XIA, Evolution Induced Catastrophe, Phys. Lett. A. Vol 185, pp.196-200, 1994.

[9] XIA, M. F., SONG, Z. Q., XU, J. B., ZHAO, K. H., and BAI, Y. L., Sample-specific Behavior in Failure Models of Disordered Media, Communication in Theoretical Physics. Vol 25, pp. 49-54, 1996.

[10] Bai Y L, Hao S W, Lu M F, et al. Damage evolution induced catastrophe and its signification in forecasting terrestrial disasters. Journal of Terraspace Science and Engineering, Vol 1 41 48, 2009.

[11] X. H. Xu, M. F. Xia, F. J. Ke and Y. L. Bai, Experimental Evidence of Critical Sensitivity in Catastrophe. Pure and Applied Geophysics, Vol 16, pp. 1751 1767, 2006.

[12] Hao S. W. Localization, Catastrophic Rupture and Critical Singularity of Heterogeneous Brittle Media. PhD thesis, Institute of Mechanics, Chinese Academy of Sciences (CAS) ,2007.

[13] $\mathrm{Yu}$ W. and Alberto M. Cuitino, Full-field measurements of heterogeneous deformation patterns on polymeric foams using digital image correlation. International Journal of Solids and Structures, Vol 39, pp. 4777 3796, 2002.

[14] Z. Sun, Jed S. Lyons and Stephen R. McNeill, Measuring Microscopic Deformations with Digital Image Correlation. Optics and Laser in Engineering, Vol 27, pp. 409 428, 1997. 